\title{
Real-time urban traffic amount prediction models for dynamic route guidance systems
}

\author{
Zilu Liang $^{1{ }^{*}}$ and Yasushi Wakahara ${ }^{1,2}$
}

\begin{abstract}
The route guidance system (RGS) has been considered an important technology to mitigate urban traffic congestion. However, existing RGSs provide only route guidance after congestion happens. This reactive strategy imposes a strong limitation on the potential contribution of current RGS to the performance improvement of a traffic network. Thus, a proactive RGS based on congestion prediction is considered essential to improve the effectiveness of RGS. The problem of congestion prediction is translated into traffic amount (i.e. the number of vehicles on the individual roads) prediction, as the latter is a straightforward indicator of the former. We thereby propose two urban traffic prediction models using different modeling approaches. Model-1 is based on the traffic flow propagation in the network, while Model-2 is based on the time-varied spare flow capacity on the concerned road links. These two models are then applied to construct a centralized proactive RGS. Evaluation results show that (1) both of the proposed models reduce the prediction error up to $52 \%$ and $30 \%$ in the best cases compared to the existing Shift Model, (2) providing proactive route guidance helps reduce average travel time by up to $70 \%$ compared to providing reactive one and (3) non-rerouted vehicles could benefit more from route guidance than rerouted vehicles do.
\end{abstract}

Keywords: Urban traffic prediction; Route guidance system; Intelligent transport system; Traffic modelling

\section{Introduction}

Urban road traffic congestion has been a global issue for many years due to rapid urbanization. Residents in cities are suffering from the most annoying side-product of urbanization every day. According to data from IBM [1], there are more than one billion cars running on all the roads around the world, and the number will double by 2020. Traffic congestion not only causes mental stress in drivers, but also leads to more severe pollution, higher gasoline consumption and huge economic loss $[2,3]$. There are three levels of solutions to urban road traffic congestion: reducing road traffic demand, shifting road traffic to other travel mode, and spatially distributing traffic to maximize the usage of traffic network capacity. Since it is not always possible to reduce the number of trips or persuade drivers to change their travel mode, distributing traffic through route guidance is considered a most feasible, effective and economic solution to urban

\footnotetext{
*Correspondence: z.liang@cnl.t.u-tokyo.ac.jp

${ }^{1}$ Department of Electrical Engineering and Information Science, Graduate School of Engineering, The University of Tokyo, 2-11-16 Yayoi, Bunkyo-ku, Tokyo 113-8658, Japan

Full list of author information is available at the end of the article
}

road traffic congestion. Consequently, the route guidance system (RGS) has attracted great research interest from the government [4], companies $[1,5,6]$ and research institutes [7] for many years. In our daily life, RGS has been widely used to facilitate driving either in unfamiliar or familiar environment through providing turn-by-turn route navigation or recommending real-time optimal route information.

The route guidance provided by RGS can be based either on prevailing real-time traffic condition (prevailing route guidance) or predicted traffic condition (predictive route guidance), and it has been widely recognized in the transportation engineering community [8] that when predictions are accurate, predictive information is generally expected to be more effective than prevailing information because predictive information accounts for the rapid change of traffic conditions spatially and temporally. Although a couple of anticipatory RGSs have been proposed in academia $[9,10]$, these systems are fundamentally reactive solutions. In other words, current route guidance systems are no more than alert systems, as they provide drivers traffic information after congestion

\section{是 Springer}

(c) 2014 Liang and Wakahara; licensee Springer. This is an Open Access article distributed under the terms of the Creative Commons Attribution License (http://creativecommons.org/licenses/by/2.0), which permits unrestricted use, distribution, and reproduction in any medium, provided the original work is properly credited. 
happens instead of proactively guiding drivers to prevent congestion from happening. Due to this strategic limitation of current RGS, the traffic prediction module in existing RGS has been mainly focusing on travel time prediction and the consistency of predicted travel time.

We argue that an RGS has the potential to bring more benefit to a transportation system if it gives proactive route guidance to related vehicles, which helps drivers detour to reduce the degree of congestion and even prevent congestion from happening. The mechanism of this kind of proactive RGS is to provide route guidance to drivers when traffic congestion is predicted. In this paper, the problem of congestion prediction is translated to the prediction of traffic amount (i.e. the number of vehicles) on a road link, as high traffic amount is a straightforward indicator of congestion. We propose traffic amount prediction models tailored for urban road links adopting two distinct modeling approaches, with the intension of laying a foundation for proactive RGS. The first model is based on the propagation of traffic flow along the successive road links on a route, while the second model is based on time-varied occupied link capacity on the concerned links. In order to evaluate the prediction accuracy of the proposed models, we run simulations in a microscopic simulator SUMO [11], conduct prediction using the proposed models, and analyze the prediction errors under the effect of varied prediction interval. The results demonstrate advantages of the proposed prediction models compared to an existing one. We then apply the prediction models to an RGS to investigate how the proactive route guidance affects the performance of a traffic network (i.e. average travel time, average travel length) and the impact of rerouting on drivers (e.g. the number of rerouted vehicles, average travel time of rerouted/nonrerouted vehicles, etc.).

The main contributions of this paper are listed below:

- We propose two novel traffic amount prediction models tailored for urban traffic networks based on two distinct microscopic modeling approaches.

- We construct a proactive RGS based on the proposed prediction models to improve the performance of RGS.

- We evaluate the prediction accuracy of our models using realistic traffic traces on real city maps, and compare their performance with a good baseline model. We demonstrate the advantage of the proposed models with respect to varied prediction interval.

- We investigated the effectiveness of proactive route guidance with respect to the performance of a traffic network on the system level and the impact of the guidance on rerouted and non-rerouted drivers.
The rest of the paper is organized as follows: In section 2, we discuss related works on RGS and traffic prediction; the proposed urban traffic amount prediction models are presented in section 3; in section 4, we construct a centralized proactive RGS based on the prediction models; in section 5 , we evaluate the prediction accuracy of the proposed models, and investigate the impact of proactive route guidance on a traffic network; in the last section, we draw the conclusions.

\section{Related work}

\subsection{Route guidance systems (RGS)}

Route guidance systems [12] were originally invented to facilitate drivers to arrive at their destinations when traveling in unfamiliar environment. Early route guidance service was limited to in-vehicle car navigation [13], which was initially used only by a small proportion of people because these systems were expensive and mainly installed in high-end cars. Moreover, first generation route guidance systems primarily compute the shortest routes on the basis of static map and do not respond to real-time traffic condition.

With the development of traffic surveillance infrastructure and communication technologies, it is possible to generate dynamic route guidance based on real-time traffic conditions $[14,15]$. The benefit of route guidance system has thereby been extended far beyond the traditional turn-by-turn navigation function. Even when drivers are traveling in familiar environment, they feel the need to use route guidance service to acquire the information on not only real-time traffic conditions and suggestions on alternative routes that avoid on-going congestion, but also road pricing, parking availability, and even entertainment facilities. Although the efficiency of route guidance is closely related to the quality of real-time traffic data, simulation study has confirmed the potential of RGS in reducing average travel time and congestion severity even with imperfect traffic information [16].

In academia, besides the research effort on the mechanism of route guidance generation, many researchers have also devoted to the study on individual drivers' responses or compliance to route guidance [17-21]. It is worth noticing that, in [21], the authors conclude from questionnaire surveys that 'it would be naive to assume that ... a guidance system could cause equipped drivers in a familiar network to take routes very different from those they would wish to take' and 'those drivers who are congestion avoiders would be more malleable than those who are time minimisers'. At the same time, most drivers have high expectations of the potential savings in time that might be gained by following route guidance for travels made in congested conditions even in familiar environment. The above fact confirmed the necessity to improve the function of RGS in terms of combating road traffic congestion, which will not 
only bring more benefit to drivers but serve as enforcement to drivers' compliance. In recognition of this need, we intend to design a dynamic RGS that helps reduce the degree of traffic congestion or even prevent congestion from happening. Specifically, our goal is to reduce average travel time in the traffic network by providing proactive route guidance that is based on short-term traffic amount (and thus congestion) prediction.

\subsection{Traffic prediction}

Intensive research effort has been made on traffic prediction in traffic engineering, with a dominant amount of work done on travel time prediction. According to the type of data a prediction model is based on, we can classify existing models into two categories: models based on historical traffic data and models based on real-time traffic data.

Most of the traditional prediction models belong to the first category, including historical average and smoothing techniques, parametric and non-parametric regression [22-24], autoregressive integrated moving average (ARIMA) [25-27], machine learning [28], fuzzy logic $[29,30]$ and neural networks [31-33]. These methods often suffer from high computational complexity either due to the stationery requirements or a large number of estimated parameters and may not be adaptive to the change in traffic patterns [34]. Smith and Demetsky [35] conducted comparisons of historical average, time-series, nonparametric regression and artificial neural network (ANN), and found that the non-parametric regression model significantly outperformed the other models and was easier to implement. Even so, non-parametric regression models require large amount of historical data and training process. Moreover, in the scenario where matches are not enough good in the historical database, the non-parametric regression may fail to output reliable prediction.

In order to improve the prediction accuracy, several models were proposed based on real-time traffic data $[36,37]$. Very recently, a traffic flow prediction method for signal-controlled city street network has been proposed in [38]. However, some input variables required by this model are usually difficult to obtain in real transportation systems. Furthermore, the speed-density fundamental diagram [39] adopted by this model may not hold in urban traffic networks, as the dependency of travel speed on the traffic flow in urban areas is not significant [40] and may demonstrate multivaluedness and instability $[41,42]$. For a single urban link, the speed on this link is not only dependent on flows on the link itself but also on other conflicting links $[43,44]$.

Despite the varying degrees of accuracy that have been achieved by these prediction models, they can hardly be effectively applied to realize dynamic route guidance that helps prevent congestion from happening. These models are fundamentally macroscopic or mesoscopic, and therefore, it is not easy to accommodate the effect of traffic lights and other traffic management measures such as dynamic route guidance and congestion pricing. Besides, some of the models are based on classic traffic flow theory that is originally established for highways and does not necessarily hold on urban links. In this paper, we adopt a microscopic modeling approach to compensate the demerits of existing modeling approaches, and develop effective traffic prediction models to facilitate congestion prediction and construct proactive RGS based on such prediction.

\section{The proposed prediction models}

\subsection{Problem definition}

The goal of our problem is to predict the traffic amount on a road link in urban traffic network based on realtime traffic information. The traffic amount on link $i$ in time interval $k$, denoted as $X_{i}(k)$, is defined as the number of vehicles on link $i$ at the beginning of time interval $k$. Although conventionally transportation researchers have been focusing on the parameter of traffic flow, or traffic volume, defined as the number of vehicles passing an observation point per unit of time (usually $1 \mathrm{~h}$ ) [45], we believe that traffic amount is a better target for our problem than traffic flow or volume, as high traffic amount is a direct indicator of traffic congestion. Besides, it is feasible to count the traffic amount on urban links, whereas the same task can hardly be done on highways.

Suppose in an urban network there is a centralized traffic control center that periodically conducts traffic amount prediction and generates route guidance based on the prediction. The control center considers the traffic network as a discrete-time system and adopts the rolling horizon approach [46] to conduct the prediction. In other words, the time horizon is divided into discrete traffic prediction time intervals whose length is $\tau$ seconds, and traffic prediction is performed repeatedly every $\tau$ seconds and at the beginning of each time interval. In practice, the traffic control center needs to carefully decide on the value of $\tau$ to ensure effective and feasible prediction. If $\tau$ is too long, the prediction output cannot facilitate timely traffic management. On the other hand, if $\tau$ is too short, the new round of prediction is not meaningful, as new traffic data will not have become available at the traffic control center. Suppose there are traffic sensors (e.g. loop detectors and probe cars) on all road links and each sensor provides a traffic data at given time interval $\tau_{A G}$, we define the short-term traffic amount prediction problem as follows:

Definition 1. Given the observed traffic data on all the links during time interval $k$, find the traffic amount on a link $i$ during time interval $k+1$. 
We adopt a microscopic modeling approach to take into consideration of the impact of traffic signal and drivers' route choice on traffic flow. Compared to conventional mesoscopic modeling based on traffic flow theory, our modeling approach can not only effectively capture the sudden change in traffic flow pattern, but easily be integrated to traffic management measures such as adaptive traffic signaling and dynamic route guidance.

Before presenting the details of the two proposed urban traffic prediction models, we first clarify the assumptions for the models here. We assume that the number of vehicles on each link at an initial time equals zero. The traffic amount is assumed to stay constant during each prediction time interval. The traffic data aggregation period interval $\tau_{A G}$ is equivalent to the prediction interval $\tau$. Moreover, we assume that the split rate of traffic flows at the intersections and the departure/arrival traffic amount on each link are obtained beforehand, e.g. via vehicle tracking $[47,48]$, vehicular route prediction [49] or even by collecting drivers' feedback on their route choice [50].

\subsection{Model-1: prediction based on spatiotemporal correlation}

Model-1 is based on the rationale that a traffic flow propagates along successive links in a traffic network, and the flow thus has spatiotemporal characteristics. It has been shown in [34] that utilizing traffic amount of the nearest upstream links helps improve the prediction accuracy, but those of distant neighbouring links do not affect it. Therefore, Model-1 considers the spatial correlation between the concerned link and each of its direct upstream links. In addition, the traffic flow may be split up at intersections depending on the predetermined destinations of vehicles and the routes selected by the drivers. Model-1 is formulated as follows:

$$
\hat{X}_{i}(k+1)=\max \left\{\hat{X}_{i, \text { calculated }}(k+1), 0\right\}
$$

and

$$
\begin{gathered}
\hat{X}_{i, \text { calculated }}(k+1)=X_{i}(k)+\hat{Q}_{i, \text { in }}(k)+X_{\mathrm{dep}, i}(k) \\
-\hat{Q}_{i, \text { out }}(k)-X_{\mathrm{arr}, i}(k) \\
\hat{Q}_{i, \text { in }}(k)=\sum_{u=1}^{U_{i}} X_{(i-1)_{u}}(k) \cdot \gamma_{(i-1)_{u} \rightarrow i}(k) \cdot \delta_{(i-1)_{u} \rightarrow i}(k) \\
\hat{Q}_{i, \text { out }}(k)=\sum_{d=1}^{D_{i}} X_{i}(k) \cdot \gamma_{i \rightarrow(i+1)_{d}}(k) \cdot \delta_{i \rightarrow(i+1)_{d}}(k)
\end{gathered}
$$

where $\hat{X}_{i}(k+1)$ is the predicted traffic amount on link $i$ in time interval $k+1, X_{i}(k)$ and $X_{(i-1)_{u}}(k)$ are the detected traffic amount on link $i$ and its $u$ th upstream in time interval $k, \hat{Q}_{i \text {,in }}(k)$ and $\hat{Q}_{i \text {,out }}(k)$ are the predicted traffic amount that enters and leaves link $i$ respectively, $X_{\mathrm{dep}, i}(k)$ and $X_{\mathrm{arr}, i}(k)$ are the departure and arrival traffic amount on link $i, \gamma_{(i-1)_{u} \rightarrow i}(k)$ is the split rate of traffic amount that travels on the $u$ th upstream of link $i$ in time interval $k$ and will enter link $i$ afterwards, $\gamma_{i \rightarrow(i+1)_{d}}(k)$ is the split rate of the traffic amount that travels on link $i$ and will enter the $d$ th downstream link in time interval $k$ afterwards. The $\delta_{(i-1)_{u} \rightarrow i}(k)$ is the adjustment factor for the traffic flow from the $u$ th upstream link to link $i$ in time interval $k$, and $\delta_{i \rightarrow(i+1)_{d}}(k)$ is the adjustment factor for the traffic flow from link $i$ to its $d$ th downstream link. The adjustment factors take into account the effect of traffic lights on traffic dynamics.

Equation 1 ensures that the predicted traffic amount is always non-negative; if the outcome of Equation 2 is negative, it will be replaced by zero. The temporal correlation between the predicted traffic amount and the current traffic amount on link $i$ is represented by Equation 2. The spatial correlations between the predicted traffic amount on link $i$ and each of the current traffic amount on neighboring links, captured by the predicted inflow and outflow traffic amount, are represented by Equations 3 and 4 , respectively. To be more specific, the split rate $\gamma_{(i-1)_{u} \rightarrow i}(k)$ and $\gamma_{i \rightarrow(i+1)_{d}}(k)$ adaptively capture the direction of traffic propagation, while the adjustment factors $\delta_{(i-1)_{u} \rightarrow i}(k)$ and $\delta_{i \rightarrow(i+1)_{d}}(k)$ adaptively capture the speed of traffic propagation under the effect of traffic lights. The adjustment factors are defined by Equations 5 and 6, where $T^{g}(k)$ is the ratio of green time of the traffic signal for the correspondent traffic flow during time interval $k$, $\hat{\mathrm{TT}_{i}}(k)$ and $\hat{\mathrm{TT}}(i-1)_{u}(k)$ are the estimated travel time on link $i$ and its $u$ th upstream link, respectively. As has been mentioned in the assumptions, we assume that the split rates are obtained beforehand. The adjustment factors are formulated as the length of the green phase of the traffic lights divided by the estimated link travel time.

$$
\begin{gathered}
\delta_{(i-1)_{u} \rightarrow i}(k)=\frac{T_{(i-1)_{u} \rightarrow i}^{g}(k)}{\hat{\mathrm{TT}}_{(i-1)_{u}}(k)} \\
\delta_{i \rightarrow(i+1)_{d}}(k)=\frac{T_{i \rightarrow(i+1)_{d}}^{g}(k)}{\hat{\mathrm{TT}}_{i}(k)}
\end{gathered}
$$

For simplicity, we estimate the link travel time using Equation 7 in the implementation of this model, though we are aware of the possibility to adopt more comprehensive travel time estimation algorithms e.g. using probe data [51].

$$
\hat{\mathrm{TT}}_{i}(k)=\frac{L_{i}}{\bar{v}_{i}(k)}
$$

where $L_{i}$ is the length of link $i$, and $\bar{v}_{i}(k)$ is the average travel speed on link $i$ in time interval $k$. Here the speed information is assumed to be obtained from loop detectors or sensors rather than calculated using the conventional speed-density-flow relationship. The reason is 
that for a road link in a city, the dependency of travel speed on the flow is not very significant, and the travel speed is mainly dependent on the type and the geometry of the link [40]. Using data from inductive loop detectors, including vehicle count and occupancy, the average speed of vehicles can be estimated from Equation 8 [52].

$$
\bar{v}_{i}(k)=\frac{N_{i}(k) \cdot L_{\mathrm{eff}}}{\tau_{\mathrm{AG}} \cdot o_{i}(k)}
$$

where $N_{i}(k)$ and $o_{i}(k)$ are the measured traffic count and occupancy (the percentage of time the detector is occupied by vehicles) on link $i$ in time interval $k$, respectively, and $L_{\text {eff }}$ is the average effective vehicle lengths (EVLs) of the traffic stream, which is the average vehicle length plus the detector length. In practice, $L_{\text {eff }}$ has been assumed to be constant; for example, the Washington State Department of Transportation uses $L_{\text {eff }}=20$ to $25 \mathrm{ft}$ [53]. If no loop detector has been installed on road links, it is still possible to estimate the real-time vehicle speed using telecommunication technologies $[54,55]$.

\subsection{Model-2: prediction based on spare road capacity}

Model-2 is based on the rationale that the inflow and outflow on the concerned link are largely determined by its spare capacity. In order to quantify the time-varied spare capacity of a road link, we first define the maximum outflow and inflow that are possible on a link in a certain time interval. The maximum outflow of a road link $i$ to its $d$ th downstream link $(i+1)_{d}$ in time interval $k$, denoted by $S_{i \rightarrow(i+1)_{d} \text {,out }}(k)$, is defined as the maximum number of vehicles that can exit link $i$ and enter link $i+1$ in this time interval. Similarly, the maximum inflow of link $i$, denoted by $S_{i, \text { in }}(k)$, is defined as the maximum number of vehicles that can enter link $i$ from all its upstream links. The maximum outflow and inflow are determined by the real-time traffic situation on the concerned link, which may vary significantly at different time intervals. The $S_{i \rightarrow(i+1)_{d} \text {,out }}(k)$ and $S_{i, \text { in }}(k)$ can be estimated by the following formulas:

$$
\begin{aligned}
& S_{i \rightarrow(i+1)_{d}, \text { out }}(k)=\frac{T_{i \rightarrow(i+1)_{d}}^{g}(k) \cdot \bar{v}_{i}(k)}{L_{v}+L_{g}} \\
& S_{i, \text { in }}(k)=\frac{\bar{v}_{i}(k)}{L_{v}+L_{g}}
\end{aligned}
$$

where $L_{v}$ and $L_{g}$ are the average vehicle length and the minimum gap between vehicles, respectively.

Accordingly, the predicted inflow and outflow is an adjustment of the maximum flows based on the real-time vehicle occupancy on the concerned links. On the basis of the above consideration, Model- 2 is formulated below.

$$
\hat{X}_{i}(k+1)=\max \left\{\hat{X}_{i, \text { calculated }}(k+1), 0\right\}
$$

and

$$
\begin{gathered}
\hat{X}_{i, \text { calculated }}(k+1)=X_{i}(k)+\hat{Q}_{i, \text { in }}(k)+X_{\text {dep }, i}(k) \\
-\hat{Q}_{i, \text { out }}(k)-X_{\text {arr }, i}(k) \\
\hat{Q}_{i, \text { in }}(k)=\min \left\{\sum_{u=1}^{U_{i}} S_{(i-1)_{u} \rightarrow i, \text { out }}(k) \cdot \gamma_{(i-1)_{u} \rightarrow i}(k)\right. \\
\left.\cdot \frac{\bar{X}_{(i-1)_{u}}(k)}{C_{(i-1)_{u}}}, S_{i, \text { in }}(k)\right\} \\
\hat{Q}_{i, \text { out }}(k)=\sum_{d=1}^{D_{i}} \min \left\{S_{i \rightarrow(i+1)_{d}, \text { out }}(k) \cdot \gamma_{i \rightarrow(i+1)_{d}}(k)\right. \\
\left.\cdot \frac{\bar{X}_{i}(k)}{C_{i}}, S_{(i+1)_{d}, \text { in }}(k)\right\} \\
\delta_{(i-1)_{u} \rightarrow i}(k)=\frac{T_{(i-1)_{u} \rightarrow i}^{g}(k)}{\operatorname{TT}_{(i-1)_{u}}(k)} \\
\delta_{i \rightarrow(i+1)_{d}}(k)=\frac{T_{i \rightarrow(i+1)_{d}}^{g}(k)}{\hat{\mathrm{TT}}_{i}(k)}
\end{gathered}
$$

where $\bar{X}_{(i-1)_{u}}(k)$ and $\bar{X}_{i}(k)$ are the average traffic amount on the $u$ th upstream link and link $i$ respectively in time interval $k, C_{(i-1)_{u}}$ and $C_{i}$ are the capacity of the $u$ th upstream link and that of link $i$. It is worth noting that the capacities used in Equations 13 and 14 are in fact the queuing capacity [56] of a road defined as the number of vehicles that can be stored on the road in a queue. When this storage capacity is exceeded the queue will spill back onto the upstreams of this road and often block intersections.

The maximum flows are calculated according to the definitions by Equations 9 and 10 .

$$
\begin{aligned}
& S_{(i-1)_{u} \rightarrow i, \text { out }}(k)=\frac{T_{(i-1)_{u} \rightarrow i}^{g}(k) \cdot \bar{v}_{(i-1)_{u}}(k)}{L_{v}+L_{g}} \\
& S_{i \rightarrow(i+1)_{d}, \text { out }}(k)=\frac{T_{i \rightarrow(i+1)_{d}}^{g}(k) \cdot \bar{v}_{i}(k)}{L_{v}+L_{g}} \\
& S_{i, \text { in }}(k)=\frac{\bar{v}_{i}(k)}{L_{v}+L_{g}} \\
& S_{(i+1)_{d} \text {,in }}(k)=\frac{\bar{v}_{(i+1)_{d}}(k)}{L_{v}+L_{g}}
\end{aligned}
$$

The main difference between Model-1 and Model-2 lies in the prediction of inflow and outflow traffic amount. Model- 1 considers the propagation of traffic on successive links, which is adaptively adjusted according to the realtime traffic conditions. In contrast, Model-2 predicts the actual inflow/outflows by taking the maximum flows that is possible on the concerned link during that time interval as reference. As is shown in Equation 13, the predicted inflow of link $i$ is the minimum of the following two values: (1) the maximum inflow of link $i$ and (2) the sum of the 
maximum outflow of the direct upstream links adjusted by the split rates and occupied road capacity. Similarly, as is shown in Equation 14, the predicted outflow of link $i$ is the sum of the minimum of the following two values: (1) the maximum inflow of a direct downstream link and (2) the maximum outflow of link $i$ adjusted by the split rates and occupied road capacity.

\section{Applying prediction models to RGS}

We apply the proposed urban traffic amount prediction models to a typical centralized RGS to construct the proactive RGS. The proposed RGS operates in three phases: (1) detecting and predicting congestion, (2) selecting vehicles for rerouting and computing alternative routes and (3) pushing route guidance to drivers. The RGS also adopts the rolling horizon approach; that is, the time horizon is divided into discrete time intervals, and the three phases are conducted at the beginning of each time interval repeatedly. It is worth noticing that the control time interval $\tau_{c}$ of the RGS is equivelent to the prediction time interval $\tau$. Each of the phases is described in detail below.

\subsection{Detecting and predicting congestion}

The centralized RGS service provider periodically collects traffic data, e.g. traffic amount on each road link $X_{i}(k)$, every $\tau$ minutes, where $\tau$ is the duration of a traffic control interval, and $k$ is the index of control interval. Based on the collected real-time traffic data, the service provider predicts $\hat{X}_{i}(k+1)$ for all road links using a traffic amount prediction model, and then detects and predicts traffic congestion using Equations 21 and 22. A link is considered to be currently congested if Equation 21 is satisfied, while a link is considered to be will-be-congested if Equation 22 is satisfied.

$$
\begin{aligned}
& \frac{X_{i}(k)}{C_{i}} \geq \alpha \\
& \frac{X_{i}(k)}{C_{i}}<\alpha \quad \text { and } \quad \frac{\hat{X}_{i}(k+1)}{C_{i}} \geq \alpha
\end{aligned}
$$

where $\alpha \in[0,1]$ is a predefined congestion threshold value.

\subsection{Selecting vehicles and computing alternative routes} When congestion is detected or predicted on a road, vehicles that satisfy the following two requirements will be selected for rerouting: (1) they are on up to the $l$-hop upstream of the congested or will-be-congested link and (2) they intend to use this link afterwards. The selection level, denoted as $l$, needs to be properly chosen to mitigate congestion without triggering secondary congestion on popular alternative routes [7]. The service provider then computes the shortest alternative route for selected vehicles using the Dijkstra algorithm based on current travel time on each road.

\subsection{Pushing route guidance to vehicles}

When the service provider completes the computation of alternative routes for all selected vehicles, it pushes the guidance to each of the vehicles. Vehicles are expected to switch to the guided alternative routes and continue their travel.

\section{Performance evaluation}

The purpose of the evaluation is to clarify the answers to the following questions

\section{With respect to the prediction models:}

- How to decide on the prediction interval $\tau$ ? How does $\tau$ affect the accuracy of the prediction models?

\section{With respect to the proactive RGS:}

- How to decide on the congestion threshold $\alpha$ ? How does $\alpha$ effect the system performance of an RGS?

- How does proactive route guidance affect the performance of a traffic network on its system level? How does control/prediction interval affect the ultimate system performance?

- How many vehicles are involved in rerouting? Is the impact of rerouting the same on rerouted and non-rerouted vehicles?

We adopt an open-source and highly portable microscopic traffic simulator, SUMO [11], to run simulations and collect real traffic data. The route guidance function is realized by employing Traffic Control Interface (TraCI) [57]. TraCI provides an access to a running road traffic simulation in a real-time mode so that we can change the route of a vehicle on the run. The default setting in SUMO 0.15 .0 is used to configure vehicles. The vehicle length is $5 \mathrm{~m}$, the minimal gap between vehicles is $2.5 \mathrm{~m}$, and the Krauss model [58] is used as car following model. We use different network topology and traffic demand depending on the purpose of the evaluation, which will be described in detail in each of the following subsections.

\subsection{Accuracy of proposed prediction models}

In order to evaluate the performance of the proposed urban traffic prediction models, we implement the models to predict traffic amount in Cologne, Germany. The real traffic amount data are collected from simulations in SUMO by applying the real data of traffic demand between 6:00 and 8:00 a.m. of a day in the 'TAPAS Cologne' Scenario [59] to the simulator. We intend to predict the traffic amount on link $23572355 \sharp 2$ that has three 
upstream links and two downstream links. The parameters of the local topology of link $23572355 \sharp 2$ are shown in Table 1.

We compare our proposed models with the existing Shift Model, which is represented by Equation 23.

$$
\hat{X}_{i}(k+1)=X_{i}(k)
$$

Since the prediction interval is small, the Shift Model is considered a very competitive prediction model for dynamic traffic networks. We employ the following measures as criteria to evaluate the accuracy of the prediction models: Mean Absolute Error (MAE) and Symmetric Mean Absolute Percent Error (SMAPE) [60]. The definitions of these measures are shown below.

$$
\begin{aligned}
& \text { MAE }=\frac{1}{K} \sum_{k=1}^{K}|\hat{X}(k)-X(k)| \\
& \mathrm{SMAPE}=\frac{1}{K} \sum_{k=1}^{K} \frac{|\hat{X}(k)-X(k)|}{X(k)+\hat{X}(k)}
\end{aligned}
$$

where $K$ is the total number of prediction intervals, $X(k)$ are the values collected from the simulations in SUMO, while $\hat{X}(k)$ are the predicted values. The reason for adopting two measures is that a combined evaluation based on both measures can compensate for the potential disadvantages of each single measure and thus provide a better picture of the errors. On the one hand, MAE is scale-dependent so that it cannot be compared across estimation series on different scales [61]; on the other hand, SMAPE is scale-independent, but it is favorable for overestimation. An observation on the difference between the trend of SMAPE and MAE could roughly indicate whether an estimation model tends to yield overestimation. It is also worth noting that we do not adopt the widely used measure Mean Absolute Percent Error (MAPE) [62] here, as this measure yields biased evaluation when real value is close to zero [62].

We conduct prediction every 10, 60, 180, and $300 \mathrm{~s}$, which are equivalent to the aggregation period of traffic data. We run the simulations five times under different seed values, and acquire five sets of real traffic data. For

Table 1 The local topology of link 23572355 $\$ 2$

\begin{tabular}{lccc}
\hline Link ID & $\begin{array}{c}\text { Length } \\
(\mathbf{m})\end{array}$ & $\begin{array}{c}\text { Max. Speed } \\
(\mathbf{m} / \mathbf{s})\end{array}$ & Relation \\
\hline $23572355 \sharp 2$ & 109 & 14 & Link $i$ \\
$-24665807 \sharp 1$ & 125 & 14 & Upstream of $i$ \\
$24665807 \sharp 0$ & 134 & 14 & Upstream of $i$ \\
$23572355 \sharp 1$ & 98 & 14 & Upstream of $i$ \\
$23585509 \sharp 0$ & 103 & 14 & Downstream of $i$ \\
$23572355 \sharp 3$ & 121 & 14 & Downstream of $i$ \\
\hline
\end{tabular}

each value of prediction interval, the prediction is conducted over each of the five sets of data and prediction errors are calculated after each prediction. The average values of the prediction errors over the five repetitions are taken as the final results, and the $95 \%$ confidence interval is also calculated.

The prediction errors MAE and SMAPE are shown in Figure 1a,b, respectively. Generally speaking, the prediction errors (both MAE and SMAPE) decrease as the prediction interval $\tau$ decreases. As the prediction is based on the collected real-time traffic information that reflects the traffic conditions in the previous $\tau$ seconds, the shorter $\tau$ is, the higher is the possibility that the current traffic conditions are close to that of $\tau$ seconds ago. Therefore, higher frequency of prediction, i.e. smaller $\tau$, has the potential to improve prediction accuracy. In reality, however, $\tau$ is bounded by the latency introduced in traffic data aggregation, transmission and processing. Therefore, short $\tau$ is desirable but not always achievable. For example, if the detected traffic data is updated every $10 \mathrm{~min}$, then $\tau$ can only be longer than $10 \mathrm{~min}$; in this case, it is meaningless if we conduct traffic prediction less than every $10 \mathrm{~min}$, as new data are not yet available.

Figure 1 shows that Model-1 has the smallest errors among the three prediction models regardless of $\tau$. It significantly reduces MAE by $52 \%$ and SMAPE by $41 \%$ compared to the baseline Shift Model in the best case ( $\tau=10 \mathrm{~s})$. Model- 2 also reduces MAE by $30 \%(\tau=10 \mathrm{~s})$ and SMAPE by $28 \%(\tau=300 \mathrm{~s}$ ) compared to the Shift Model in the best cases. In the studied scenario, Model-1 gains its maximum advantage when $\tau=10 \mathrm{~s}$, which is in the same magnitude as the link travel time. As the prediction interval further increases, the advantage of Model-1 decreases, whereas the advantage of Model-2 increases. When $\tau=300 \mathrm{~s}$, the accuracy of the two proposed models is very close. We can infer that Model-1 may work better when $\tau$ is on the same magnitude as link travel time, while Model-2 may be more suitable for longer $\tau$.

In practice, the aggregation period of traffic data ranges from 20 or $30 \mathrm{~s}$ [63] to $5 \mathrm{~min}$. The prediction model should be chosen depending on the data aggregation period. For example, if the traffic data is aggregated in less than $1 \mathrm{~min}$, Model-1 should be used to perform the prediction; otherwise, Model-2 would be a better candidate to yield accurate prediction. In addition, it is worthy of mentioning that the prediction accuracy could be influenced by other factors, such as the characteristics of traffic demand, the topology of the traffic network, the route choice decision made by drivers with or without guidance, the configuration of traffic signal, etc. Hence, the ultimate requirement on the prediction accuracy could be greatly dependent on the specific applications. We also confirmed that the proposed models are not biased [64]. 


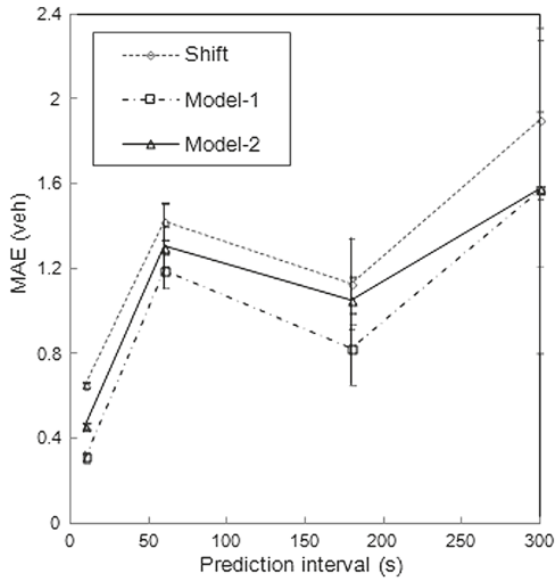

a



b

Figure 1 Prediction errors versus prediction interval $\boldsymbol{\tau}$. (a) MAE (b) SMAPE. Model-1 has the best prediction accuracy and reduces MAE and SMAPE by up to $52 \%$ and $41 \%$ respectively compared to the Shift Model in the best case ( $\tau=10$ s). Model-2 also reduces MAE and SMAPE by up to $30 \%(\tau=10 \mathrm{~s})$ and SMAPE by $28 \%(\tau=300 \mathrm{~s}$ ) compared to the Shift Model in the best cases. Reducing $\tau$ may improve prediction accuracy, but short $\tau$ is not always achievable as it is bounded by the latency of traffic data collection and data processing at the control center.

\subsection{Performance of RGS with traffic prediction}

In order to investigate the benefit of traffic prediction to RGS, we implement a typical RGS incorporated with the proposed traffic prediction models in SUMO. The map of a small proportion of London traffic network shown in Figure 2 was exported from OpenStreetMap [65]. The network includes a total of 3,002 links and 332 intersections. The exported map is then converted into required format using the tool NETGEN provided in SUMO. In the simulation, 954 vehicles are generated in total. One vehicle is generated every second between $[0,1,000] \mathrm{s}$ and each vehicle randomly selects an Origin-Destination pair. Each run of simulation last $5,000 \mathrm{~s}$ to ensure that all the vehicles would have reached their destinations before the simulation terminates.

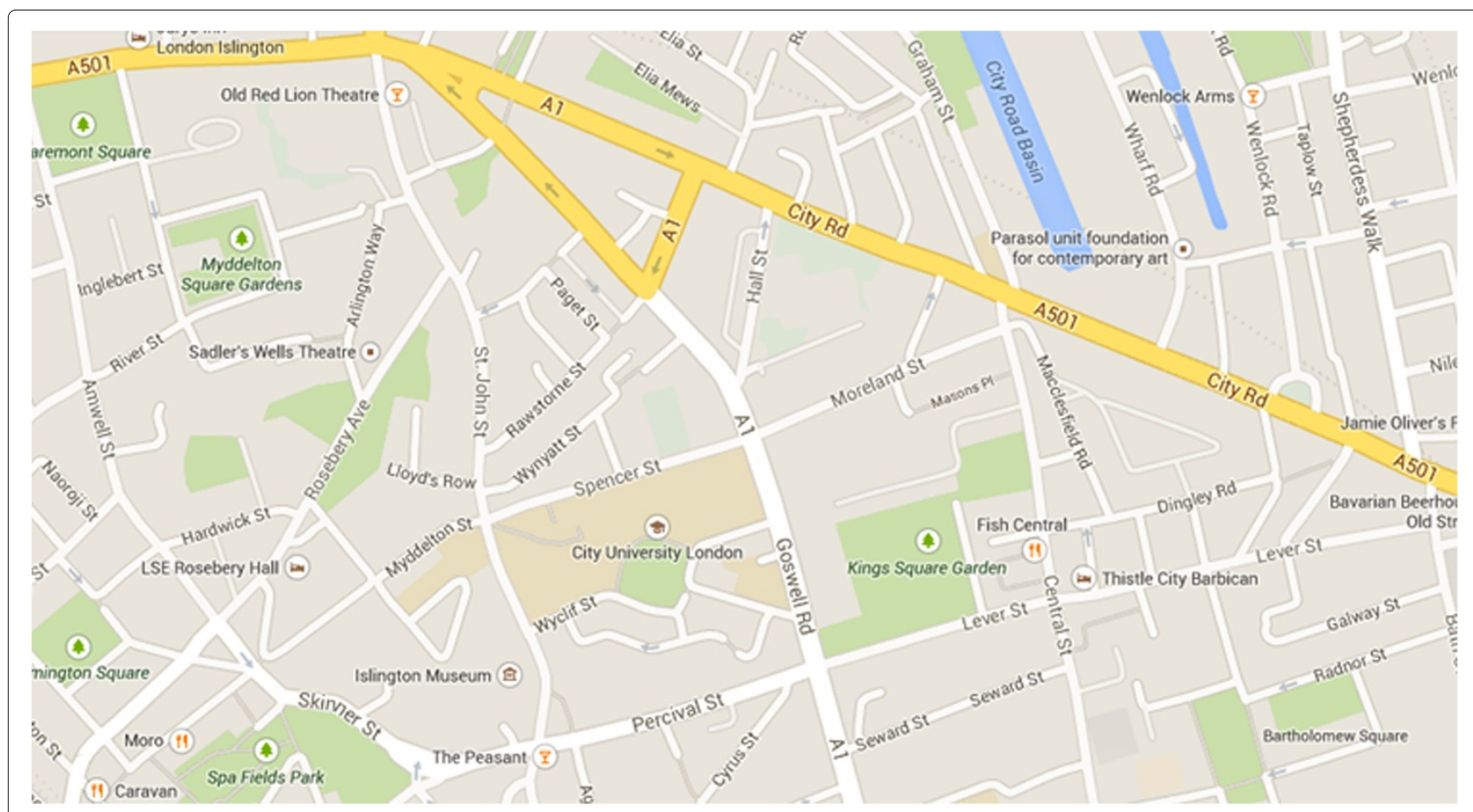

Figure 2 Topology of traffic network. A portion of London traffic network is used as the network topology in the simulations. 
We compare the performance of the following five cases.

- RGS+Model-1: A proactive RGS that generates route guidance based on traffic prediction using Model-1.

- RGS+Model-2: A proactive RGS that generates route guidance based on traffic prediction using Model-2.

- RGS+Model-shift: A proactive RGS that generates route guidance based on traffic prediction using the existing Shift Model.

- RGS: A reactive RGS that generates route guidance based on current traffic condition without prediction.

- No-RG: No route guidance is provided; thus no rerouting is performed.

We run each simulation five times under different seed values, and take the average values as the final results. In the figures, we also indicate $95 \%$ confidence intervals. The selection level $l$ is set to 3 , as it produces good results with moderate computation [7]. The penetration rate is $100 \%$; that is, all vehicles are subscribed to the route guidance service and thus can periodically receive route guidance. The compliance rate is set to $100 \%$ so that all the drivers are supposed to follow the guidance. The RGS generates route guidance every $\tau$ second, which is equivalent to the traffic prediction interval.

We first analyze the performance of the RGS under the impact of the congestion threshold $\alpha$ that is set to three fixed values $0.6,0.7$ and 0.8 . In order to evaluate the operating efficiency of the route guidance, we use average travel time as the level-of-service measure. Compared with other similar measures such as travel speed, travel time is not only intuitive to travelers, but also can be easily interpreted in economic terms, which is critical to quantifying the cost and benefit of transportation investments.
The results of $R G S+$ Model-1, RGS+Model-2 and $R G S+$ Model-shift under different values of $\alpha$ are shown in Figure 3a,b,c, respectively. In each of the three cases, the impact of $\alpha$ is not obvious from the statistical point of view, as the confidence intervals for three threshold values are considerably overlapped. However, it also shows that $\alpha=0.7$ has the potential to produce the most competitive results. Therefore, the value of $\alpha$ is set to 0.7 in the hereafter evaluations. It is worth noticing that the value of $\alpha$ should be tuned according to the scenario.

With respect to the prediction/control interval $\tau$, we set it to $30,60,180$ and $300 \mathrm{~s}$ to investigate its effect on the system performance. The simulation results of average travel time is shown in Figure 4. Firstly, we confirmed that rerouting does help drivers arrive earlier, as the travel time is more than twice when no rerouting is performed. Moreover, the average travel time increases as $\tau$ increases, which indicates that increasing the frequency of traffic control may lead to more efficient traffic operation due to timely response to traffic dynamics.

Figure 4 also demonstrates the advantage of RGS incorporated with traffic congestion prediction, where the average travel time under proactive route guidance is much shorter than that under a reactive one. Route guidance based on traffic prediction leads to maximum $70 \%$ reduction in average travel time compared to that based on current traffic condition. However, the gap between the former and the latter decreases a bit as the control/prediction interval increases. The contribution of traffic prediction to travel time reduction also depends on the prediction model. Compared to the existing Shift Model, both of the proposed models can further reduce up to $14 \%$ of the average travel time in the best cases. It is interesting to notice that the average travel length, which

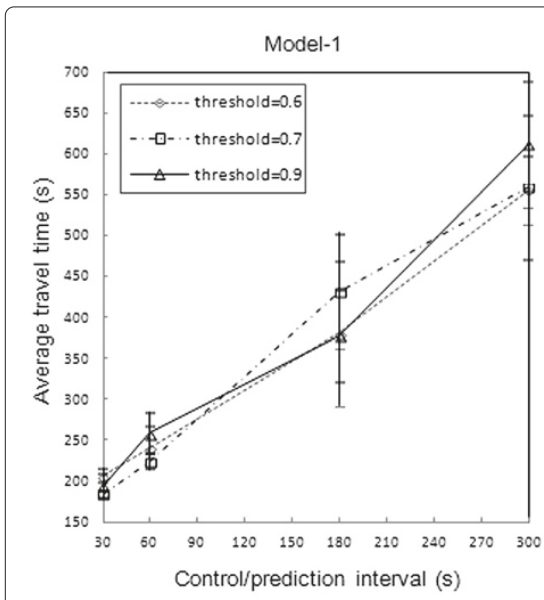

a

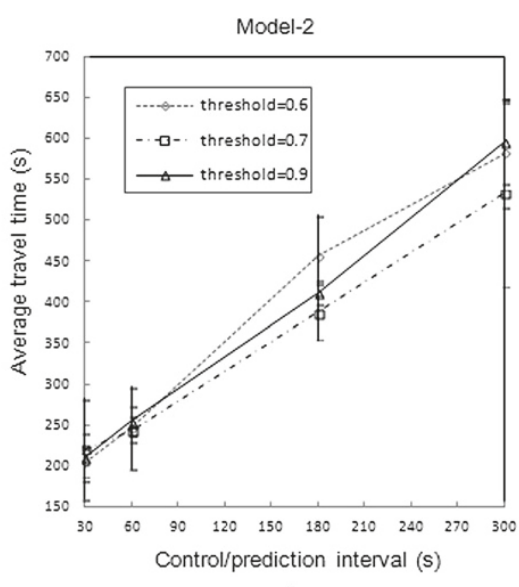

b

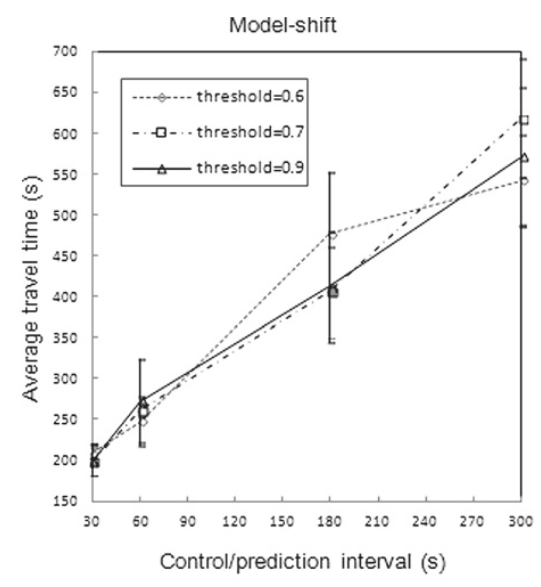

C

Figure 3 The effect of congestion threshold $\alpha(\mathbf{a}, \mathbf{b}, \mathbf{c})$. The impact of congestion threshold is not statistically obvious; however, the threshold value 0.7 has the potential to produce the most competitive outcome. 


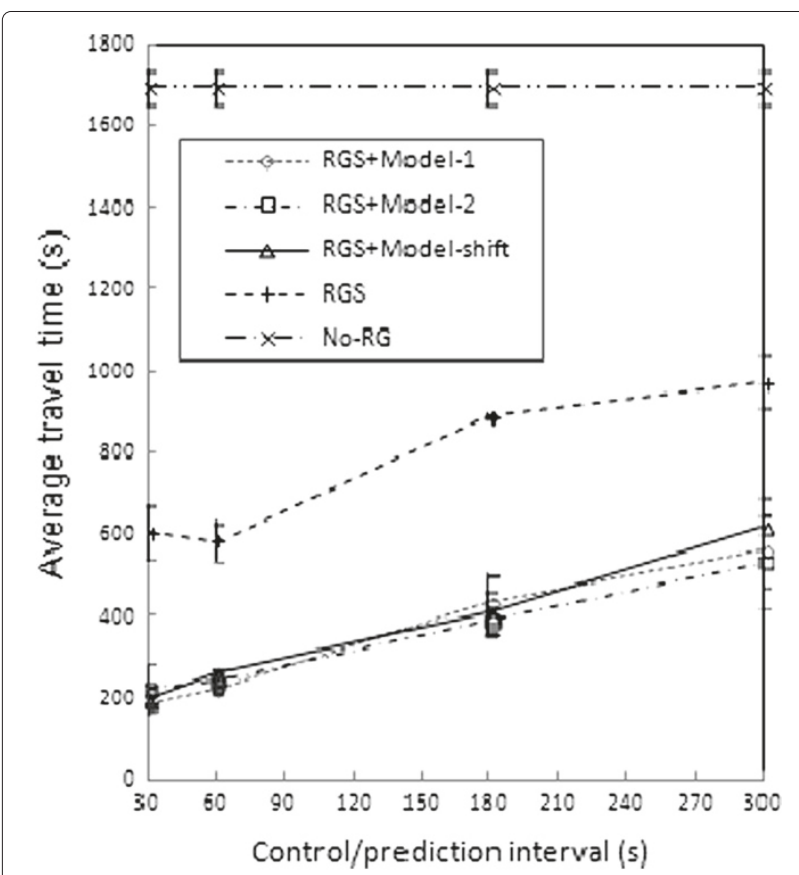

Figure 4 Average travel time versus prediction/control interval $\boldsymbol{\tau}$. Congestion prediction contributes to travel time reduction in the proposed RGS. The advantage is more obvious as $\tau$ decreases. is plotted in Figure 5, is longer if the route guidance is provided. It seems that an RGS helps drivers to save travel time at the sacrifice of traveling on longer routes. From another angle, we can infer that the fastest routes are not necessarily the shortest; traveling on longer routes could lead to earlier arrival.

In order to further understand the impact of route guidance on rerouted vehicles and non-rerouted vehicles, we also plot the average travel time for each of the two categories of vehicles in Figure 6. Since the average travel time produced by the reactive RGS is always much longer than that of the proposed proactive one, we do not include the results of the former in the figure in order to have a clear comparison among the proactive ones with different prediction models. Simulation results also show that both rerouted and non-rerouted vehicles are benefited from traffic prediction, as the average travel time for both categories of vehicles is much shorter under predictive route guidance than that under reactive route guidance. However, the average travel time of non-rerouted vehicles is shorter in comparison with rerouted vehicles. An explanation to this phenomenon could be that the route-switch of rerouted vehicles helps mitigate the on-going congestion or reduce the possibility of potential congestion on originally shortest routes, and thus facilitates smooth travel of non-rerouted vehicles on their original routes.

Figures 7 and 8 demonstrate the total number of rerouted vehicles and the average number of reroutings

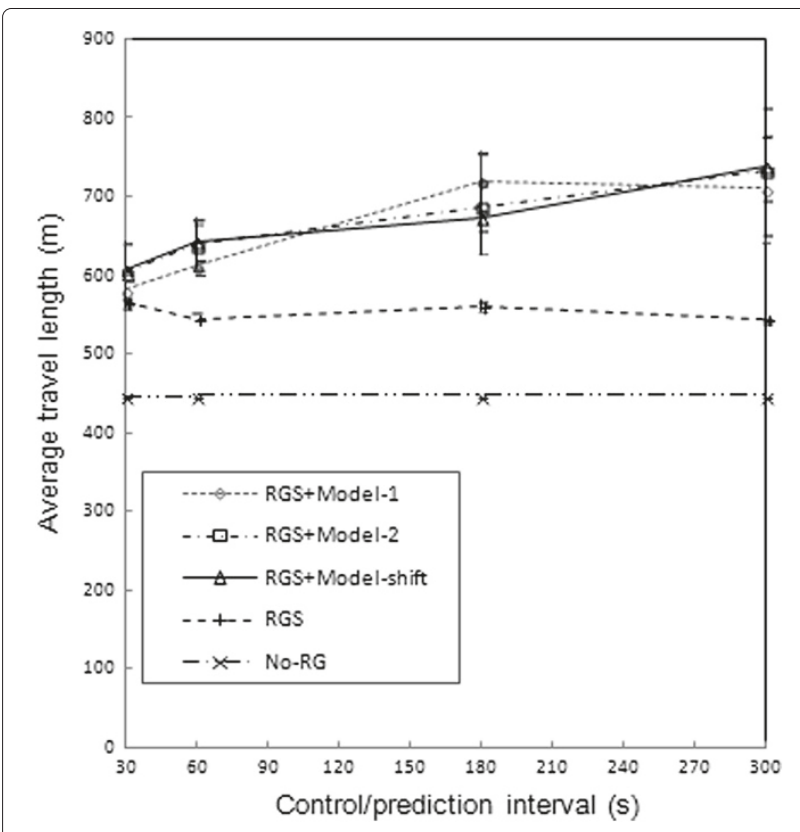

Figure 5 Average travel length versus prediction/control interval $\boldsymbol{\tau}$. Route guidance leads to longer travel length. As $\tau$ increases, the average length of the conventional RGS stays almost constant, whereas that of the proposed predictive RGS increases probably due to the longer routes used to avoid predicted congestion.

for each rerouted vehicle, respectively. It is interesting that the number of vehicles involved in rerouting does not change much as the control/prediction interval increases, and reactive route guidance affects slight less vehicles than predictive one does. On the other hand, the average number of reroutings for each rerouted vehicle decreases as the control/prediction interval increases. This is reasonable because longer interval means less frequent interaction between the route guidance service provider and the drivers. Moreover, individual vehicles are rerouted more times under reactive route guidance than predictive guidance. We can infer that proactive route guidance can give preventive response to traffic conditions and thus less times of reroutings for each vehicle.

\section{Conclusions}

In this paper, we proposed two urban traffic amount prediction models based on the propagation of traffic flow and the spare road capacity, respectively, for applying the proposed models to a route guidance system (RGS) to reduce average travel time. We evaluated the prediction accuracy of the proposed models by comparing their performance with the Shift Model under varied prediction interval using the real data collected in the traffic simulator SUMO. The results demonstrated that both models significantly reduce prediction error up to $52 \%$ and $30 \%$ in the best cases compared to the existing Shift Model. 


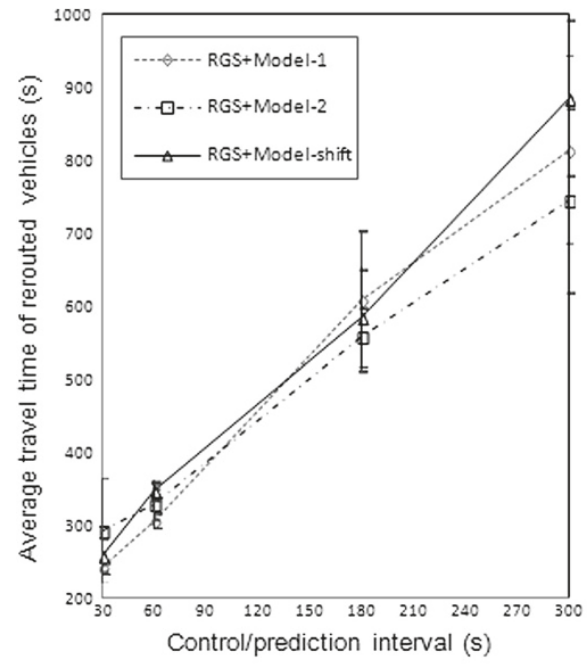

a

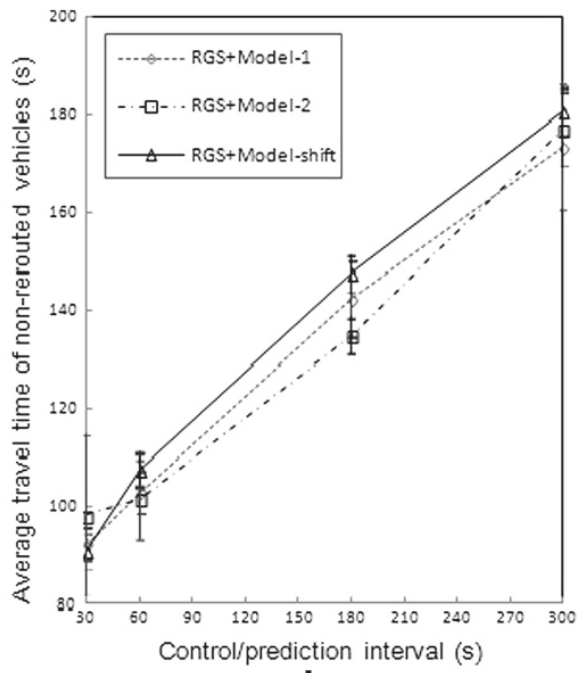

b

Figure 6 Average travel time versus prediction/control interval $\boldsymbol{\tau}$ among (a) rerouted vehicles and (b) non-rerouted vehicles. Both rerouted and non-rerouted vehicles are benefited from traffic prediction, as the average travel time for both categories of vehicles is much shorter under predictive route guidance than that under reactive route guidance. However, non-rerouted vehicles could enjoy more benefit than rerouted ones.

In addition, we found that the performance of Model-1 peaks when the prediction interval is in the same magnitude as the link travel time, while Model-2 demonstrates superiority when the prediction interval is longer. We also evaluated the impact of proactive route guidance by comparing the performance of an RGS with traffic amount prediction (proactive route guidance) to that of an RGS without prediction (reactive route guidance). Simulation

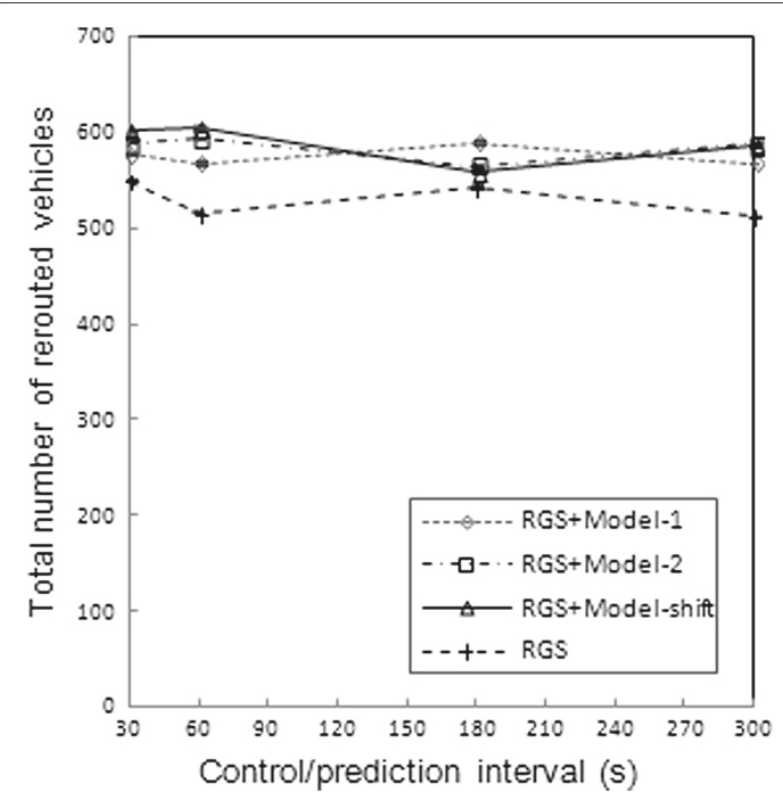

Figure 7 Total number of rerouted vehicles. The number of vehicles involved in rerouting does not change much as $\tau$ increases, and the conventional route guidance affects slightly less vehicles than predictive one does. results confirmed that the proactive route guidance helps greatly reduce average travel time by up to $70 \%$ compared to the reactive ones, and the proposed traffic prediction models can further reduce average travel time by up to $14 \%$ in comparison with the existing Shift Model.

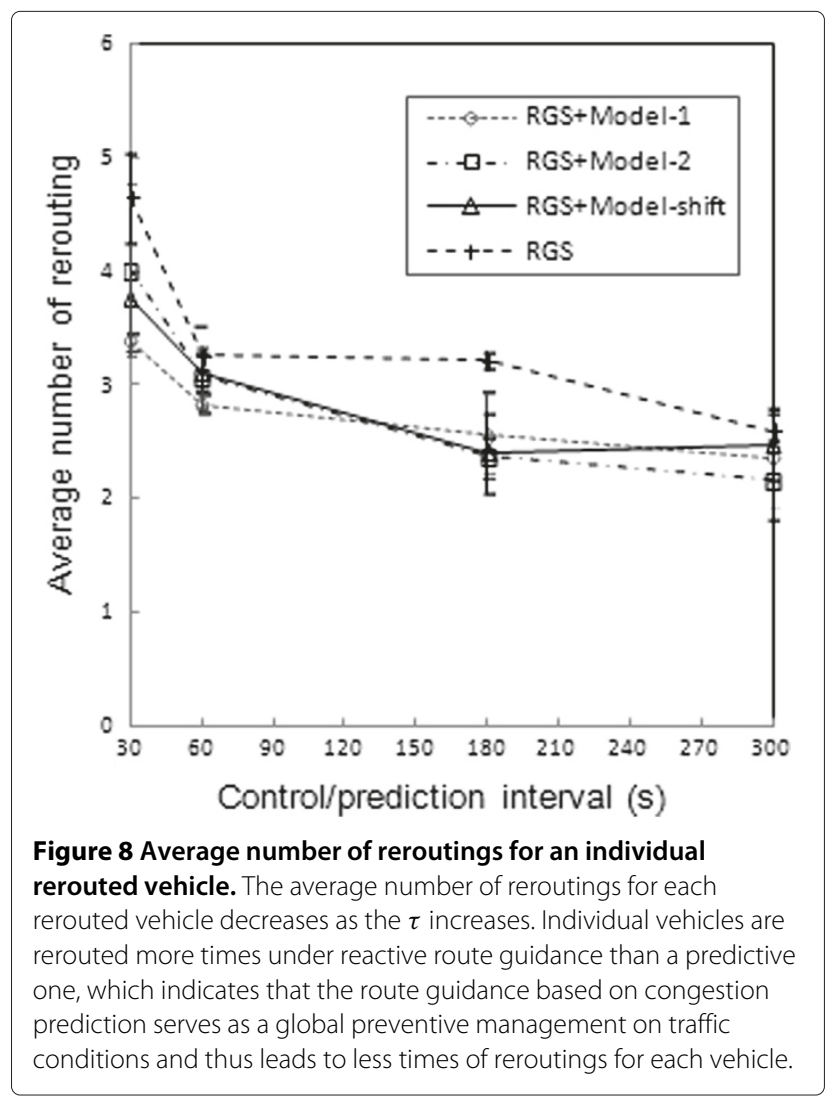


Moreover, proactive route guidance leads to less number of reroutings for each rerouted vehicle. We also discovered that non-rerouted vehicles could benefit more from route guidance than rerouted vehicles do. In the next step, we intend to propose more efficient route scheduling and routing mechanism in RGS with the objective of pushing the system performance to its optimal.

\section{Competing interests}

The authors declare that they have no competing interests.

\section{Acknowledgements}

The authors would like to thank the anonymous reviewers for their valuable comments and suggestions on this paper.

\section{Author details}

${ }^{1}$ Department of Electrical Engineering and Information Science, Graduate School of Engineering, The University of Tokyo, 2-11-16 Yayoi, Bunkyo-ku, Tokyo 113-8658, Japan. ${ }^{2}$ Information Technology Center, The University of Tokyo, 2-11-16 Yayoi, Bunkyo-ku, Tokyo 113-8658, Japan.

Received: 31 January 2014 Accepted: 30 April 2014

Published: 22 May 2014

\section{References}

1. IBM Smart Traffic (2010). http://www.ibm.com/smarterplanet/us/en/ traffic_congestion/ideas. Accessed May 2014

2. D Schrank, B Eisele, T Lomax, TTI's 2012 Urban mobility report. (Texas A\&M Transp. Inst., College Station, TX, USA, 2012)

3. S Grant-Muller, J Laird, Costs of Congestion: Literature Based Review of Methodologies and Analytical Approaches. (Scottish Executive, Edinburgh 2007)

4. DA Rosen, FJ Mammano, R Favout, An electronic route-guidance system for highway vehicles. IEEE Trans. Vehicular Technol. 19(1), 143-152 (1970)

5. Tomtom (2013). http://www.tomtom.com. Accessed May 2014

6. Google Maps. https://www.google.com/mobile/maps/. Accessed May 2014

7. J Pan, IS Popa, K Zeitouni, C Borcea, Proactive vehicular traffic rerouting for lower travel time. IEEE Trans. Vehicular Technol. 628, 3551-3568 (2013)

8. J Dong, HS Mahmassani, CC Lu, How reliable is this route? Predictive travel time and reliability for anticipatory traveler information systems. Transportation Res. Rec. 1980(1), 117-125 (2006)

9. R Claes, T Holvoet, D Weyns, A decentralized approach for anticipatory vehicle routing using delegate multiagent systems. IEEE Trans. Intell. Transportation Syst. 12(2), 364-373 (2011)

10. K Chen, SE Underwood, Research on anticipatory route guidance, in Proceedings of Vehicle Navigation and Information Systems Conference, vol. 2 (Warrendale, 1991), pp. 427-439

11. D Krajzewicz, J Erdmann, M Behrisch, L Bieker, Recent development and applications of SUMO - simulation of urban mobility. Int. J. Adv. Syst. Meas. 5(3\&4), 2012

12. M Khanjary, SM Hashemi, Route guidance systems: review and classification, in Proceedings of 6th Euro American Conference on Telematics and Information Systems (EATIS) (Valencia, 2012), pp. 1-7

13. DE Boyce, Route guidance systems for improving urban travel and location choices. Transportation Res. 22A(4), 275-281 (1988)

14. I Skog, P Handel, In-car positioning and navigation technologies: a survey. IEEE Trans. Intell. Transportation Syst. 10(1), 4-21 (2009)

15. TChoe, A Skabardonis, P Varaiva, Freeway performance measurement system (PeMS): an operational analysis tool. Transportation Res. Rec. 1811, 67-75 (2002)

16. Z Liang, J Polak, Y Wakahara, Simulation studies on the benefit of route guidance generated from imperfect traffic data. IEICE Tech. Rep. 112(470), 37-42 (2013)

17. Y Zhou, J Wu, The research on drivers' route choice behavior in the presence of dynamic traffic information, in Proceedings of IEEE Intelligent Transportation Systems Conference (Toronto, 2006), pp. 1304-1309
18. M Tsavachidis, Aggregate analysis of driver response to collective route guidance and implications for system control, in Proceedings of Tenth International Conference on Road Transport Information and Control (London, 2000), pp. 17-21

19. PW Bonsall, M Joint, Driver compliance with route guidance advice: the evidence and its implications, in Proceedings of Vehicle Navigation and Information Systems Conference (Warrendale, 1991), pp. 47-59

20. RW Allen, AC Stein, TJ Rosenthal, D Ziedman, JF Torres, A Halati, A human factors simulation investigation of driver route diversion and alternate route selection using in-vehicle navigation systems, in Proceedings of Vehicle Navigation and Information Systems Conference (Warrendale, 1991), pp. 9-26

21. PW Bonsall, T Parry, Drivers' requirements for route guidance, in Proceedings of Third International Conference on Road Traffic Control (London, 1990), pp. 1-5

22. H Markovic, BD Basic, H Gold, F Dong, K Hirota, Gps data based non-parametric regression for predicting travel time in urban traffic networks. Sci. Traffic Transport. 22(1), 1-13 (2010)

23. $\mathrm{CH}$ Wu, JM Ho, DT Lee, Travel-time prediction with support vector regression. IEEE Trans. Intell. Transportation Syst. 5(4), 276-281 (2004)

24. X Zhang, JA Rice, Short-term travel time prediction. Transportation Res. Part C. 11, 187-210 (2003)

25. G Comert, A Bezuglov, An online change-point-based model for traffic parameter prediction. IEEE Trans. Intell. Transportation Syst. 14(3), 1360-1369 (2013)

26. W Min, L Wynter, Real-time road traffic prediction with spatio-temporal correlations. Transportation Res. Part C 19, 606-616 (2011)

27. J Xia, M Chen, W Huang, A multistep corridor travel-time prediction method using presence-type vehicle detector data. J. Intell. Transportation Syst: Technol. Plann. Oper. 15(2), 104-113 (2011)

28. J Rzeszotko, SH Nguyen, Machine learning for traffic prediction. Fundam. Inf. 119(3-4), 407-420 (2012)

29. VP Vijayan, B Paul, Multi objective traffic prediction using type-2 fuzzy logic and ambient intelligence, in Proceedings of 2010 International Conference on Advances in Computer Engineering (ACE) (Egypt, 2010), pp. 309-311

30. L Li, WH Lin, H Liu, Type-2 fuzzy logic approach for short-term traffic forecasting. IEE Proc. Intell. Transport Syst. 153(1), 33-40 (2006)

31. S Dunne, B Ghosh, Weather adaptive traffic prediction using neurowavelet models. IEEE Trans. Intell. Transportation Syst. 14(1), 370-379 (2013)

32. E Mazloumi, G Rose, G Currie, S Moridpour, Prediction intervals to account for uncertainties in neural network predictions: methodology and application in bus travel time prediction. Eng. Appl. Artif. Intell. 24(3), 534-542 (2011)

33. CPIJ van Hinsbergen, A Hegyi, JWC van Lint, HJ van Zuylen, Bayesian neural networks for the prediction of stochastic travel times in urban networks. IET Intell. Transport Syst. 5(4), 259-265 (2011)

34. B Ghosh, B Basu, M O'Mahony, Multivariate short-term traffic flow forecasting using time-series analysis. IEEE Trans. Intell. Transportation Syst. 10(2), 246-254 (2009)

35. BL Smith, BM Williams, RK Oswalsd, Comparison of parametric and nonparametric models for traffic flow forecasting. Transportation Res. Part C 10(4), 303-321 (2002)

36. Y Wang, M Papageorgiou, A Messmer, Real-time freeway traffic state estimation based on extended kalman filter: a case study. Transportation Sci. 4(2), 167-181 (2007)

37. M Chen, SIJ Chien, Dynamic freeway travel-time prediction with probe vehicle data: link based versus path based. Transportation Res. Rec 1768, 157-161 (2001)

38. QJ Kong, Y Xu, S Lin, D Wen, F Zhu, Y Liu, UTN-model-based traffic flow prediction for parallel-transportation management system. IEEE Trans. Intell. Transportation Syst. 14(3), 1541-1547 (2013)

39. MJ Lighthill, GB Whitham, On kinematic waves II: a theory of traffic flow on long crowded roads. Proc. Roy. Soc. Lond. Ser. A 229(1178), 317-345 (1955)

40. WR Mcshane, RP Roess, Traffic Engineering. (Prentice Hall, Inc, 1990)

41. N Geroliminis, B Boyaci, The effect of variability of urban systems characteristics in the network capacity. Transportation Res. Part B 46, 1607-1623 (2012)

42. CF Daganzo, W Gayah, EJ Gonzales, Macroscopic relations of urban traffic variables: bifurcations, multivaluedness and instability. Transportation Res. Part B 45, 278-288 (2011) 
43. LG Willumsen. 2nd edn, ed. by DA Hensher, Button J K, Travel networks, in Handbook of Transport Modelling, vol. 1 (Elsevier Oxford, UK, 2008), pp. 203-220

44. Z Liang, Y Wakahara, Speed-volume relationship model for speed estimation on urban roads in intelligent transportation systems, in Proceedings of the 23rd International Conference on System Engineering (Las Vegas, 2014)

45. Detection Technology: for IVHS-Volume 1, Final Report Addendum (1995). http://ntl.bts.gov/DOCS/96100/ch02/ch02.html. Accessed May 2014

46. SAZRXI-pN TL Pan, Short-term traffic state prediction based on temporal-spatial correlation. IEEE Trans. Intell. Transportation Syst. 14(3), 1242-1254 (2002)

47. S Sivaraman, MM Trivedi, Integrated lane and vehicle detection, localization, and tracking: a synergistic approach. IEEE Trans. Intell. Transportation Syst. 14(2), 906-917 (2013)

48. J Hu, A Razdan, JC Femiani, M Cui, P Wonka, Road network extraction and intersection detection from aerial images by tracking road footprints. IEEE Trans. Remote Sensing. 45(12-2), 4144-4157 (2007)

49. G Xue, Z Li, H Zhu, Y Liu, Traffic-known urban vehicular route prediction based on partial mobility patterns, in Proceedings of 15th International Conference on Parallel and Distributed Systems (Shenzhen, 2009), pp. 369-375

50. JM Choi, Multi-touch based standard UI design of car navigation system for providing information of surrounding areas. Design, User Exp. Usability User Exp. Novel Technological Environ. 8014, 40-48 (2013)

51. A Hofleitner, R Herring, P Abbeel, A Bayen, Learning the dynamics of arterial traffic from probe data using a dynamic Bayesian network. IEEE Trans. Intell. Transportation Syst. 13(4), 1679-1693 (2012)

52. P Athol, Interdependence of certain operational characteristics within a moving traffic stream. Highway Res. Record. 72, 58-87 (1965)

53. JM Ishimaru, ME Hallenbeck, Flow evaluation design technical report. Washington Dept. Transp., Seattle, WA, Tech. Rep. WA-RD 13(4), 1679-1693 (1999)

54. RH Liou, YB Lin, YL Chang, HN Hung, NF Peng, MF Chang, Deriving the vehicle speeds from a mobile telecommunications network. IEEE Trans. Intell. Transportation Syst. 14(3), 1208-1217 (2013)

55. BY Lin, CH Chen, CC Lo, A traffic information estimation model using periodic location update events from cellular network. Commun. Comput. Inf. Sci. 135, 72-77 (2011)

56. DA Hensher, KJ Button, Handbook of Transport Modelling. (Elsevier, 2008)

57. Traffic Control Interface (TraCl) in SUMO (2013). http://sourceforge.net/ apps/mediawiki/sumo/index.php?title=TraCl. Accessed May 2014

58. S Krauss, Microscopic modelling of traffic flow: investigation of collision free vehicle dynamics. PhD thesis, University of Cologne (1998)

59. "TAPAS Cologne"i Scenario (2013). http://sumo-sim.org/doc/current/ docs/userdoc/Data/Scenarios.html. Accessed May 2014

60. BE Flores, A pragmatic view of accuracy measurement in forecasting. Omega Int. J. Manage. Sci. 14, 93-98 (1986)

61. RJ Hyndman, Another look at forecast accuracy metrics for intermittent demand. Int. J. Appl. Forecasting 4, 43-46 (2006)

62. JS Armstrong, Principles of Forecasting: A Handbook for Researchers and Practitioners. (Springer, New York, 2001)

63. ML Hazelton, Estimating vehicle speed from traffic count and occupancy data. J. Data Sci. 2, 231-244 (2004)

64. Z Liang, Y Wakahara, ed. by City traffic prediction based on real-time traffic information for intelligent transportation systems, in Proceedings of 13th International Conference on ITS Telecommunications (ITST) (Finland 2013), pp. 378-383

65. Open Street Map, (2012). http://www.openstreetmap.org Accessed May 2014

\section{doi:10.1186/1687-1499-2014-85}

Cite this article as: Liang and Wakahara: Real-time urban traffic amount prediction models for dynamic route guidance systems. EURASIP Journal on Wireless Communications and Networking 2014 2014:85. 